\title{
A formação dos agentes de combate às endemias no contexto da dengue: análise documental das políticas de saúde
}

\author{
Qualification of the health community workers in the context of the \\ dengue: a document analysis of the health policies
}

\section{La formación de los agentes de las enfermedades endémicas en el contexto de la dengue: análisis documental de las políticas de salud}

Janete Gonçalves Evangelista | janetege@fiocruz.br

Fiocruz, Centro de Pesquisas René Rachou. Belo Horizonte, Brasil.

Tácia Maria Pereira Flisch | taciaflisch@gmail.com

Fiocruz, Centro de Pesquisas René Rachou. Belo Horizonte, Brasil.

Denise Nacif Pimenta | denise.pimenta@cpqrr.fiocruz.br

Fiocruz, Centro de Pesquisas René Rachou. Belo Horizonte, Brasil.

\section{Resumo}

A dengue é uma arbovirose de grande impacto na saúde pública. Fundamentando-se na importância dos agentes de combate às endemias (ACE) para prevenção e controle da doença, este artigo objetiva analisar a temática da formação dos ACE nas políticas públicas com base em pesquisa qualitativa, documental e descritiva de fontes primárias dos acervos eletrônicos do Ministério da Saúde e Ministério da Educação, disponibilizados até fevereiro de 2016. No total, 14 documentos foram analisados conforme análise de conteúdo. Os resultados indicam escassez de publicações sobre as políticas de formação profissional no contexto da dengue. Embora exista uma política incipiente visando à formação dos ACE, ainda não há regulamentação do seu trabalho técnico. É necessário discutir e aprofundar quem é esse trabalhador e os motivos pelos quais a regulamentação da formação não consta nas pautas das políticas de controle da dengue e seus vetores.

Palavras-chave: recursos humanos em saúde; formação profissional em saúde; agente de combate às endemias; análise documental; dengue. 


\begin{abstract}
Dengue is an arbovirus of great public health impact. Based on the importance of community health workers (CHW) in the prevention and control of dengue, this article aims to analyze the professional qualification of CHW in public health policies related to dengue; using a qualitative descriptive document analysis of the primary sources of electronic documents of the Ministry of Health and the Ministry of Education, available online until February 2016. In total, 14 documents were analyzed based on content analysis. The results show few publications about training policies in the context of the dengue. Even though there is an incipient policy for CHW qualification, there is no regulation of their technical work. It is necessary to discuss who are these workers and the reasons why the regulation of CHW training does not appear in the agenda of dengue control policies and its vectors.
\end{abstract}

Keywords: human resources in health; human resources training for health; community health workers; document analysis; dengue.

\title{
Resumen
}

El dengue es un arbovirus de alto impacto en la salud pública. Basándose en la importancia de los agentes para combatir las enfermedades endémicas en la prevención y control de la dengue, este artículo tiene como objetivo analizar el tema de la formación de esos agentes en las políticas públicas, utilizando un estudio cualitativo, descriptivo y documental de fuentes primarias de investigación de documentos electrónicos del Ministerio de Salud y del Ministerio de Educación, disponibles hasta febrero de 2016. En total, 14 documentos fueron analizados de acuerdo con el análisis de contenido. Los resultados indican la escasez de publicaciones sobre políticas de formación profesional en el contexto del dengue. Aunque exista una política incipiente a la formación de los mencionados agentes, no existe una regulación de su trabajo técnico. Es necesario discutir y profundizar quién es ese trabajador y las razones por las que la regulación de la formación no aparece en las agendas de las políticas de control del dengue y sus vectores.

Palabras clave: recursos humanos para la salud; formación profesional en salud; agente para combatir las enfermedades endémicas; análisis de documentos; dengue.

INFORMAÇÕES DO ARTIGO

Contribuição dos autores: Janete Gonçalves Evangelista trabalhou na concepção e redação do artigo; Tácia Maria Pereira Flisch auxiliou na construção metodológica e interpretação dos dados; Denise Nacif Pimenta coordenou todas as etapas do trabalho, bem como a revisão crítica do texto.

Declaração de conflito de interesses: Eu, Janete Gonçalves Evangelista, autora do manuscrito intitulado "A Formação dos Agentes de Combate às Endemias no Contexto da Dengue: análise documental das políticas de saúde" declaro que dentro dos últimos 05 anos e para o futuro próximo não possuo conflito de interesse de ordem: pessoal, comercial, acadêmica, política e financeira no manuscrito. Declaro também que todo apoio financeiro e material recebido para o desenvolvimento da pesquisa que resultou na elaboração do manuscrito estão claramente informados no texto do mesmo.

Fontes de financiamento: Centro de Pesquisas René Rachou/Fiocruz Minas

Considerações éticas: estudo foi aprovado pelo Conselho de Ética em Pesquisas do Centro de Pesquisas René Rachou/Fiocruz; cadastrado com o número CAAE: 50521315.7.0000.5091.

Histórico do artigo: Submetido: Submetido: 01.dez.2016 | Aceito: 01.fev.2017 | Publicado: 31.mar.2017

Apresentação anterior: A formação dos agentes de combate às endemias (ACE) no contexto das arboviroses: análise documental das políticas de saúde. III Simpósio Brasileiro de Doenças Negligenciadas/Universidade Federal de Lavras.

Licença CC BY-NC atribuição não comercial. Com essa licença é permitido acessar, baixar (download), copiar, imprimir, compartilhar, reutilizar e distribuir os artigos, desde que para uso não comercial e com a citação da fonte, conferindo os devidos créditos de autoria e menção à Reciis. Nesses casos, nenhuma permissão é necessária por parte dos autores ou dos editores. 


\section{Introdução}

Nos últimos anos, a dengue tem sido uma das arboviroses de maior impacto econômico, social e de saúde pública nas comunidades onde ocorre. Na América Latina, a doença tem se disseminado em surtos cíclicos. No Brasil, a transmissão vem acontecendo de forma continuada desde 1986. As epidemias de dengue têm sido um desafio permanente para o SUS. O aumento significativo de demanda nas unidades de saúde exige capacidade de organização dos gestores e de políticas que garantam tanto o acesso e a qualidade na assistência quanto a execução de medidas de controle do vetor capazes de reduzir a intensidade da transmissão $0^{1}$.

Presencia-se no país recentemente a inserção de novas arboviroses como a entrada da Chikungunha em 2010 e do vírus Zika em 2014; transmitidas pelo mesmo vetor da dengue, o Aedes Aegypti (da espécie Ae. aegypti). A inserção dessas arboviroses passa, então, a demandar do poder público ainda mais recursos e esforços no sentido de buscar o controle desse vetor e a prevenção dessas doença.

Apesar da incontestável importância dos agentes de combate às endemias (ACE) na prevenção e controle da dengue, percebe-se que a pouca produção acadêmica que se debruça sobre esse ator não tem aprofundado a temática de forma satisfatória. De forma inversa, as pesquisas e publicações sobre os agentes comunitários de saúde (ACS) apresentam uma produção considerável ${ }^{2-7}$. Os ACE não costumam ser alvo de estudos que se debruçam sobre as políticas públicas relacionadas à formação e ao processo de suas práticas de trabalho. Assim, a produção acadêmica sobre os ACE ainda é restrita.

Entretanto, no campo de conhecimento denominado como trabalho, educação e saúde, é possível encontrar publicações que buscam a compreensão do papel desses atores sociais no contexto da vigilância em saúde; do conhecimento deles acerca do saneamento e ambiente ${ }^{8}$; das práticas educativas dos ACE no controle de zoonozes ${ }^{9}$; do seu processo de formação $0^{10}$ e identidade profissional ${ }^{11}$. Tais estudos contribuem de forma significativa para a compreensão da temática. Contudo, ainda há necessidade de se ampliar e aprofundar o foco de análise sobre os ACE, em especial com relação às questões como: formação, educação e processos de trabalho dos mesmos.

No contexto atual das cíclicas epidemias de dengue e, ainda, a avassaladora inserção das novas arboviroses, é necessário identificar e compreender como estão se apresentando as políticas de saúde com foco na formação desses profissionais. Assim, quais seriam as políticas públicas relacionadas à formação dos ACE no contexto da dengue? Como essas políticas de prevenção e controle da doença vêm incorporando a temática da formação profissional dos ACE?

Assim, a problemática deste estudo centra-se na análise das políticas de formação direcionada aos ACE no Brasil, a partir de documentos oficiais do Ministério da Saúde e do Ministério da Educação.

\section{Políticas públicas de formação em saúde}

Entende-se como política de saúde a resposta social (ação ou omissão) de uma organização (como o Estado) diante das condições de saúde dos indivíduos e das populações e seus determinantes, bem como em relação à produção, distribuição, gestão e regulação de bens e serviços que afetam a saúde humana e o ambiente. Política de saúde abrange questões relativas ao poder em saúde (politics), bem como as que se referem ao estabelecimento de diretrizes, planos e programas de saúde (policies).

Educação e saúde articulam-se como políticas públicas no âmbito do Ministério da Saúde, pois este é o órgão oficial responsável por formalizar e gerir a formação de recursos humanos para o SUS. Portanto, essa formação estrutura-se como política ao ser formulada e implementada, tanto no ensino superior quanto na educação profissional de nível médio, por meio da Secretaria de Gestão do Trabalho e da Educação na Saúde (SGTES). Segundo consta na Constituição Federal de 1988, em seu artigo 200, compete ao SUS, além 
de outras atribuições: "III - ordenar a formação de recursos humanos na área de saúde; V - incrementar em sua área de atuação o desenvolvimento científico e tecnológico"12.

Com efeito, é fundamental compreender as relações entre educação e saúde como um conjunto de campos de práticas articulados na dinâmica social, numa perspectiva histórica. Nos argumentos de Hochman, no campo da saúde pública a história pode contribuir para "incorporar uma perspectiva social de longa duração na formação e nas atividades dos profissionais de saúde" ${ }^{\prime 13}$.

Nesses termos, pressupõe-se que a "política é, sobretudo, uma atividade transformadora do real, da história"14. E, ainda, que a atividade política institucional do Estado é um conjunto de respostas às necessidades da vida social desenvolvidas pelos homens em sua história, assim como a organização da vida coletiva e o atendimento de objetivos comuns.

A política de saúde deve ser tratada como uma política social, ou seja, uma política que está voltada para a reprodução dos indivíduos e das coletividades. Esta abordagem tem como objetivo entender que, como as demais políticas sociais, a política de saúde está sujeita a múltiplos determinantes, muitas vezes contraditórios ${ }^{15}$.

Assim como a saúde, a educação e o trabalho apresentam-se como alguns desses determinantes ${ }^{16}$. Nesses termos, torna-se pertinente buscar uma melhor compreensão sobre as políticas de formação no âmbito geral da saúde e, em específico, no contexto da dengue.

\section{O agente de combate às endemias}

O trabalhador da saúde que atua diretamente na prevenção e no controle de endemias, no âmbito do SUS é o agente de combate às endemias. Os ACE são trabalhadores da saúde que compõem as equipes de zoonoses em todo território nacional. Somente a partir de 2010, sob a orientação do Ministério da Saúde, em alguns municípios brasileiros, esse trabalhador passou a integrar também as equipes de saúde da família ${ }^{17}$. No contexto da dengue, sua atuação profissional vincula-se basicamente à prática cotidiana de busca e eliminação dos focos de reprodução dos mosquitos.

No entanto, o escopo do seu processo de trabalho engloba muito mais atividades do que somente o controle e eliminação dos criadouros. De acordo com a Lei $n^{0} 11.350 / 2006^{18}$, a função do ACE envolve o desenvolvimento de atividades no âmbito da vigilância, na prevenção e no controle de doenças e na promoção da saúde, conforme as diretrizes do SUS. Portanto, além das atribuições que o faz ser conhecido popularmente como um 'mata-mosquito', o ACE é também incumbido de atuar no controle de outras endemias presentes no território nacional, tais como: esquistossomose, febre amarela, leptospirose, malária, doença de chagas, entre outras no campo da vigilância em saúde. Constata-se assim, que o conteúdo de seu trabalho é múltiplo e vasto. Fato esse que implica em uma necessidade de formação mais consistente em todas as instâncias onde atua.

No exercício prático de seu trabalho cotidiano, o ACE exerce também atividades de educação em saúde, ao orientar a população sobre os cuidados com o meio ambiente, no sentido de prevenir e controlar endemias. Segue abaixo, na Figura 1, um fluxograma que busca situar o ACE no cenário institucional da vigilância em saúde no SUS. 


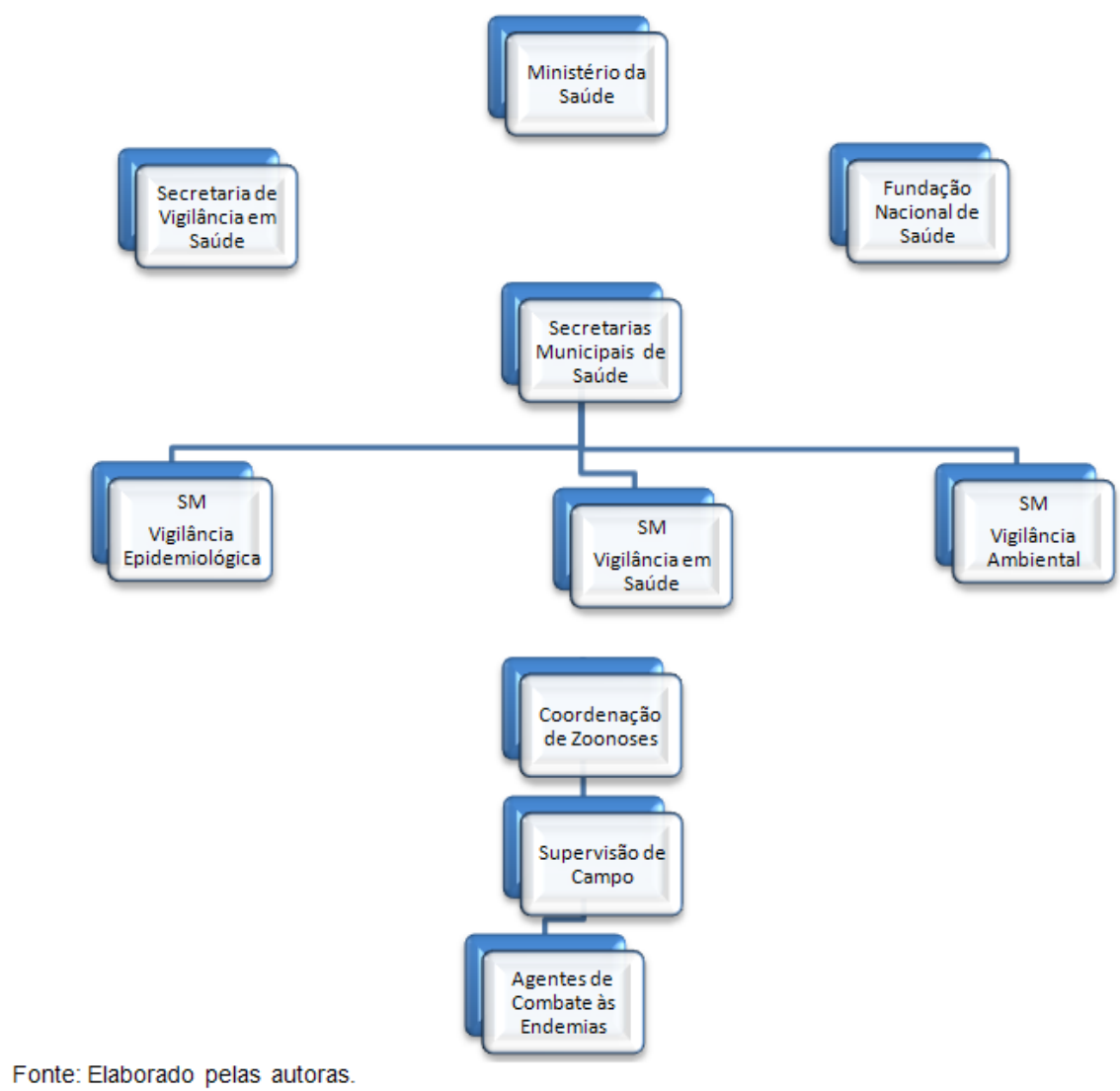

Figura 1 - O agente de combate às endemias no contexto do SUS

Ao analisar o fluxograma descrito na Figura 1, ele apresenta a inserção do ACE em relação aos entes federados e à organização geral das secretarias de saúde. Esse fluxograma é variável em cada município, dependendo da sua capacidade e organização interna. Em decorrência da descentralização das ações advinda do processo de implantação do SUS retratadas nas NOB-SUS 91 e 96 $6^{19}$, em alguns municípios, o que ficou na 'prática' é que a zoonose acabou por não se fixar de forma regular em todos os municípios brasileiros. Em algumas localidades, a vigilância é de competência da vigilância epidemiológica, em outros da vigilância em saúde, alguns na vigilância ambiental. Há ainda aqueles municípios que não possuem nenhuma dessas secretarias em seu organograma. Assim, a coordenação de zoonoses nesses municípios, acabou por vincular-se diretamente à Secretaria de Saúde.

O esforço de incorporação gradativa dos ACE, por parte do Ministério da Saúde, às equipes de Saúde da Família visa à participação no planejamento e programação das ações. Nesse contexto, é importante que as atividades dos ACS e dos ACE sejam desempenhadas de forma integrada e complementar; ambos devem assumir a corresponsabilidade pelo controle das doenças e conjugar suas atividades de forma a potencializar e melhorar a efetividade das ações ${ }^{17}$. Os ACS têm como funções: o diagnóstico demográfico e sociocultural da comunidade; ações de educação para a saúde; registro em saúde; estímulo à participação da comunidade nas políticas de saúde; visitas domiciliares periódicas; e a participação em ações de fortalecimento de ações e políticas de promoção da saúde e outras políticas que promovam a qualidade de vida ${ }^{18}$. É importante destacar que as duas categorias de trabalhadores atuam exclusivamente no âmbito do SUS e têm como responsabilidades a prevenção de doenças e a promoção da saúde. Por não ser foco da problemática deste artigo, a profissão do ACS não será pormenorizada.

Recentemente a profissão dos ACE foi incluída na Classificação Brasileira de Ocupações (CBO), organizada pelo Ministério do Trabalho e Emprego. Sua ocupação encontra-se na CBO como integrante da família "Trabalhadores em serviços de promoção e apoio à saúde" ${ }^{20}$, juntamente com os agentes 
comunitários de saúde, atendente de enfermagem, parteira leiga, visitador sanitário, agente indígena de saúde, agente indígena de saneamento e socorristas.

Além dessa inserção, foi promulgada em 2006, a Lei $11.350^{18}$ que estabelece o piso salarial e determina o exercício das atividades do ACE e do ACS. Apesar dessa discreta conquista, trata-se ainda de uma profissão que mantém vínculos precarizados de trabalho, com contratos terceirizados ou via CLT. Assim, a também precária organização da categoria ainda não conseguiu regulamentar de forma sólida essa profissão. As consequências de uma regulamentação consolidada são muitas para uma determinada atividade, e dentre elas podem-se destacar: status, sindicalização, identidade profissional ${ }^{21}$, além de outros aspectos que acabam por estruturar uma profissão. Somente em 2014, foi fixado o piso salarial para esse trabalhador. A Lei $12.994^{22}$ promulgada pela Casa Civil, além de instituir o piso, define também as diretrizes para o plano de carreira dessa profissão e dos agentes comunitários de saúde.

No âmbito da Educação Profissional, as diretrizes para a formação dos ACE se encontram no Guia Pronatec de cursos de formação inicial e continuada e no Catálogo Nacional de Cursos Técnicos. Entretanto, na prática observa-se que são poucas as reais ofertas no país de cursos de formação e treinamento direcionados para os ACE.

\section{Caminhos metodológicos}

Adotou-se como estratégia metodológica o levantamento e análise descritiva de documentos públicos, sob a premissa da pesquisa qualitativa ${ }^{23}$. Assim, a metodologia escolhida foi a da análise documental ${ }^{24-25} \mathrm{em}$ conjunto com a análise de conteúdo ${ }^{26-27}$.

A análise documental é considerada como um importante recurso para análise de políticas públicas e, por meio da interpretação de documentos, se identificam convergências, divergências e contradições nos discursos presentes entre os mesmos e a prática em saúde ${ }^{28}$.

Assim, foi realizada uma busca não sistematizada dos principais documentos oficiais disponíveis online sobre as políticas brasileiras de prevenção e controle da dengue, bem como a formação dos ACE nos acervos eletrônicos do Ministério da Saúde (MS) e do Ministério da Educação (Mec). Foram utilizados os seguintes descritores na estratégia de busca: Agente de Combate às Endemias E Dengue E Educação Profissional em Saúde E; Formação em Saúde E Capacitação em Saúde E Formação Técnica E Capacitação de Recursos Humanos. Utilizou-se o motor de busca do Google, os sistemas de busca dos próprios sites dos ministérios, além de busca manual nos respectivos espaços virtuais. A busca foi realizada em documentos disponibilizados online até fevereiro de 2016.

Como critério de inclusão dos documentos optou-se por aqueles que tratassem da formação profissional dos ACE e do tema da dengue. Além disso, os documentos deveriam ser originários das fontes oficiais da área da saúde e da educação, a saber, o Ministério da Saúde e o Ministério da Educação durante o recorte temporal especificado acima.

Fez-se uma primeira leitura flutuante do material. Logo após, foi realizada uma organização e classificação dos documentos e estabelecidos os seguintes critérios de exclusão: documentos sobre a dengue destinados a um público não relacionado aos $\mathrm{ACE}$; e os documentos que não mencionavam os $\mathrm{ACE}$ em todo o seu conteúdo foram excluídos. No Quadro 1, apresentam-se os documentos levantados e analisados conforme seu título, instituição e data de publicação. 
Quadro 1 - Acervo documental analisado

\begin{tabular}{|c|c|c|c|}
\hline Cód & Título do documento & Instituição & $\begin{array}{c}\text { Data de } \\
\text { publicação }\end{array}$ \\
\hline 01 & $\begin{array}{l}\text { Guia Pronatec de Cursos de Formação Inicial e Continuada } \\
\text { ou Qualificação Profissional: Agente de Combate a } \\
\text { Endemias }^{29}\end{array}$ & Ministério da Educação & 2016 \\
\hline 02 & O Agente Comunitário de Saúde no Controle da Dengue ${ }^{30}$ & Ministério da Saúde & 2009 \\
\hline 03 & $\begin{array}{l}\text { Dengue, esquistossomose, hanseníase, malária, tracoma, } \\
\text { tuberculose }^{31}\end{array}$ & Ministério da Saúde & 2008 \\
\hline 04 & $\begin{array}{l}\text { Diretrizes Nacionais para a Prevenção e Controle de } \\
\text { Epidemias de Dengue }^{32}\end{array}$ & Ministério da Saúde & 2009 \\
\hline 05 & Programa Nacional de Controle da Dengue ${ }^{33}$ & Ministério da Saúde & 2002 \\
\hline 06 & $\begin{array}{l}\text { Programa Nacional de Controle da Dengue - Amparo Legal } \\
\text { à Execução das Ações de Campo - imóveis fechados, } \\
\text { abandonados ou com acesso não permitido pelo morador }{ }^{34}\end{array}$ & Ministério da Saúde & 2006 \\
\hline 07 & $\begin{array}{l}\text { Técnico em Vigilância em Saúde - Diretrizes e Orientações } \\
\text { para a Formação (Profaps) }{ }^{35}\end{array}$ & Ministério da Saúde & 2011 \\
\hline 08 & $\begin{array}{l}\text { Dengue, Instruções para Pessoal de Combate ao Vetor: } \\
\text { Manual de Normas Técnicas }{ }^{36} \text {. }\end{array}$ & Ministério da Saúde & 2001 \\
\hline 09 & NotaTécnica No 2/2011/IOC-Fiocruz/Diretoria37 & Fiocruz/MS & 2011 \\
\hline 10 & NotaTécnica No 3/2014/IOC-Fiocruz/Diretoria ${ }^{38}$ & Fiocruz/MS & 2014 \\
\hline 11 & $\begin{array}{l}\text { Projeto Pedagógico do Curso de Formação Inicial e } \\
\text { Continuada ou Qualificação Profissional em Agente de } \\
\text { Combate às Endemias na modalidade presencial no âmbito } \\
\text { do Pronatec Eixo Tecnológico: Ambiente e Saúde/IFRN }{ }^{39}\end{array}$ & Ministério da Educação & 2013 \\
\hline 12 & $\begin{array}{l}\text { Catálogo Nacional de Cursos Técnicos e Tecnológicos/ } \\
\text { Agentes de Vigilância em Saúde }{ }^{40}\end{array}$ & Ministério da Educação & 2016 \\
\hline 13 & $\begin{array}{l}\text { Formação Inicial e Continuada/Agentes de Saúde IF } \\
\text { Paraná - Pronatec }{ }^{41}\end{array}$ & Ministério da Educação & 2012 \\
\hline 14 & $\begin{array}{l}\text { Programa de Formação de Agentes Locais em Vigilância } \\
\text { em Saúde (Proformar) }\end{array}$ & Ministério da Saúde & 2001 \\
\hline \multicolumn{3}{|c|}{ Total analisado } & 14 \\
\hline
\end{tabular}

Fonte: Elaborado pelas autoras (2017).

Posteriormente, durante uma segunda leitura do material levantado e triado, foram criadas tabelas no Excel para realização da análise dos documentos segundo o método de análise de conteúdo. As categorias de análises foram constituídas de acordo com os eixos detalhados no Quadro 2.

Após essa etapa, com o objetivo de interpretação e análise descritiva do material coletado, realizou-se a análise de conteúdo conforme as categorias de análise. 
Quadro 2 - Categorias de análise dos documentos

\begin{tabular}{|l|l|}
\hline \multicolumn{1}{|c|}{ Categoria de análise } & \multicolumn{1}{c|}{ Detalhamento } \\
\hline Estrutura e formatação & $\begin{array}{l}\text { Título, órgão emissor, ano de publicação, tipologia, assunto, público-alvo, autoria, } \\
\text { editora/cidade, no de páginas, anexos, atividades/exercícios, indicação de } \\
\text { estudos/atividades complementares, indicação de leitura, presença de referências } \\
\text { e glossário. }\end{array}$ \\
\hline Conteúdo & $\begin{array}{l}\text { Referência ao ACE, denominação do ACE, linguagem, abordagem do tema, } \\
\text { adequação científica, adequação ao público-alvo, tópicos abordados (estrutura } \\
\text { geral), tópicos abordados (relacionados à formação) e referencial teórico. }\end{array}$ \\
\hline Imagem & Autoria, localização, quantidade, qualidade, tipologia, título, legenda e escala. \\
\hline Referências bibliográficas & Presença ou não, adequação às normas e padronização, recorte temporal. \\
\hline
\end{tabular}

Fonte: Elaborado pelas autoras (2017).

\section{Resultados e discussão}

Inicialmente, foram levantados 21 documentos. A partir dos critérios de exclusão utilizados, no total, foram analisados 14 documentos. Estes foram analisados de forma descritiva, segundo os eixos de análise descritos no Quadro 2. Foram levantados dez documentos do Ministério da Saúde e quatro do Ministério da Educação, publicados entre 2001 e 2016.

Entre as fontes pesquisadas, um documento de natureza secundária foi recolhido com o intuito de suprir a ausência de um importante documento primário, identificado ao longo da pesquisa e recorrentemente mencionado nessas fontes. Esse documento permitiu recuperar parte relevante do conteúdo oficial original do Programa de Formação de Agentes Locais (Proformar), neste caso, contemplado por perspectivas analíticas diversas que contribuíram para a compreensão do documento primário e possibilitaram sua inevitável inclusão na presente análise. Portanto, a inclusão de tal fonte na análise documental se justifica pelo fato de o programa ter sido uma importante ação política do Ministério da Saúde. Ocorreu entre os anos de 2004 e 2007 em 26 dos 27 estados brasileiros e formou mais de 30.000 agentes de vigilância em saúde ${ }^{42}$.

As tipologias dos documentos levantados foram: programas de controle da dengue; programas de educação profissional; normas técnicas; cartilhas; diretrizes de formação em vigilância em saúde; catálogos de cursos; materiais didáticos; manuais e diretrizes para prevenção e controle da dengue; programas e projetos pedagógicos de cursos para agentes de vigilância em saúde e para os ACE. A maioria foi editada em Brasília e o restante no Rio de Janeiro, Paraná e Natal. A maioria contém anexos e complementos. Somente três documentos incluem glossário e dois se abstêm de referências bibliográficas. O número de páginas variou entre 36 e 251. Seis documentos apresentam material iconográfico que será analisado em publicação futura.

Com relação ao conteúdo, nos documentos que tratavam das endemias de uma forma geral, pouco espaço foi dado ao tema da dengue. A única cartilha (02) sobre a dengue encontrada não era direcionada ao ACE e sim aos ACS. Já a maioria do material analisado tinha como público-alvo os trabalhadores das secretarias de vigilância em saúde ou epidemiológica de uma forma mais ampla.

Chamou atenção a grande variedade de terminologias utilizadas para nomear os ACE. Em muitas situações e num mesmo documento, eles eram citados sob diferentes termos, tais como: Agente de Combate às Endemias, Agente de Endemias, Agente de Controle de Endemias, Agente de Saúde, Agente de Saúde Pública, Agente Sanitário, Agente Estatal, Agente Público, Agente da Vigilância Epidemiológica. Esse fato é um dos problemas enfrentados pelo ACE na configuração de sua identidade profissional, tanto na literatura quanto na formação de políticas públicas em saúde. 
Quanto à linguagem, ficou evidente uma preponderância de linguagem técnica, com termos mais específicos, pautados numa lógica biomédica. Por se tratar de materiais que poderiam ser utilizados como fonte de informação, uma linguagem mais próxima da realidade dos ACE poderia ser mais adequada ao seu perfil. Nas demais fontes, a linguagem configurou-se de forma mais acessível e didática, principalmente nos materiais de cursos.

De modo geral, pôde-se observar que o tema da formação - apesar de estar presente na maioria dos documentos - não se configurava como política estrutural de longa duração. Tratava-se de programas e ações mais específicos e pontuais, ou seja, políticas conjunturais. Tal enfoque superficial pode ter graves consequências, pois conforme Fonseca é necessário "recuperar as concepções de determinação social do processo saúde-doença" 4 . O mesmo argumento é sugerido por Pimenta quando afirma que "as condições sociais que originam as chamadas doenças da pobreza não são modificadas"45.

Nas fontes analisadas, a política de controle de vetores se destacou, sobrepondo-se a uma política mais consolidada de educação profissional. No documento 03, foram observadas algumas contradições e contrassensos intrigantes. Apesar de apresentar um extenso material formativo com conteúdo sobre a dengue, não consta na bibliografia nenhuma referência específica do tema. E, ainda, na mesma bibliografia analisada observou-se um número expressivo de publicações destinadas aos ACS e nenhuma direcionada ao ACE.

No documento 04, destaca-se - dentro do contexto da dengue, na Atenção primária em saúde - a promoção da capacitação dos profissionais de saúde para classificação de risco, diagnóstico, manejo clínico e assistência ao paciente com dengue. Já para os ACS, havia indicação de realização de ações de prevenção e controle junto à comunidade. Percebe-se que, nesse e em alguns outros documentos, há um investimento de inserção dos ACS no controle da dengue, seja em ações de assistência ou mesmo no controle do vetor. Há também um incentivo para que o trabalho do ACS se aproxime dos preceitos da educação em saúde. Entretanto, esses mesmos aspectos não foram identificados nas diretrizes do trabalho do ACE.

O documento 05 foi considerado como primordial para concepção das políticas direcionadas à prevenção e ao controle da dengue. Foi elaborado em 2002 especificamente para orientar o trabalho das equipes de zoonoses que lidam diretamente nas operações de campo. Além de outros componentes importantes para o trabalho do ACE, o Componente 7 do Plano Nacional de Controle da Dengue (PNCD) é voltado estritamente para a capacitação de recursos humanos. Seu objetivo é orientar o processo formativo dos trabalhadores da área "das três esferas de governo, para maior efetividade das ações nas áreas de vigilância epidemiológica, entomológica, assistência ao doente e operações de campo"33.

Entretanto, percebeu-se que as ações dos ACE descritas não são consideradas como ações de educação em saúde no PNCD. Se fossem, os ACE poderiam ter sido incluídos no componente 6, que trata das ações de educação e mobilização social, bem como no componente 4. Este último orienta ações de integração com a atenção básica. De acordo com o PNCD, o recurso destinado para a formação dos trabalhadores foi de pouco mais que nove milhões de reais.

Quanto ao documento de Amparo Legal (06), publicado posteriormente ao PNCD, o objetivo foi orientar legalmente as ações da vigilância sobre a proteção das liberdades e garantias individuais e o controle sanitário.

Segundo Batistella, a categoria profissional da vigilância é constituída por "trabalhadores dispersos em várias nomenclaturas, vinculações institucionais, relações e práticas de trabalho distintas”. Ou seja, esse quadro conceitual, normativo e mesmo nominativo teria surgido como fruto de uma multiplicidade e fragmentação do campo da vigilância (sanitária, epidemiológica, saúde ambiental e saúde do trabalhador). Pode-se responsabilizar ainda essa dispersão em função da "descentralização das responsabilidades e ações da área entre as instâncias de governo" ${ }^{\circ}$.

Já no documento do Ministério da Educação (13), aparece o curso de formação inicial e continuada na modalidade presencial do Programa Nacional de Acesso ao Ensino Técnico e ao Emprego/Pronatec. Esse programa configura-se como uma importante política de educação profissional. É destinado especificamente 
ao ACE e sua consequente certificação como agente de combate às endemias. Mesmo sendo um curso de formação mais direcionado, não consta nenhuma referência específica ao tema da dengue.

Em 2016, o Mec publicou a $3^{\text {a }}$ edição do Catálogo Nacional de Cursos Técnicos/CNCT (12) como parte de uma política de desenvolvimento e de valorização da educação profissional e tecnológica de nível médio. Os cursos vinculados à área da saúde integram o eixo tecnológico "Ambiente e Saúde". O destinado à Vigilância em Saúde é apresentado no CNCT com uma carga horária mínima de 1.200 horas, assim como os demais cursos do eixo. No entanto, mesmo instituído, só há um estado no Brasil onde o curso é ofertado, o estado do Rio de Janeiro.

No ano de 2009, a Secretaria de Gestão do Trabalho e da Educação na Saúde (SGTES) do Ministério da Saúde, a partir do Programa de Formação de Profissionais de Nível Médio para a Saúde (Profaps), toma a formação do técnico em Vigilância em Saúde como um de seus focos de atuação, promovendo encontros técnicos e oficinas de discussão sobre essa formação e sua organização curricular. O resultado desse trabalho foi publicado em 2011 em um livro intitulado Técnico em Vigilância em Saúde: diretrizes e orientações para a formação (07), onde se mantém o direcionamento dado pelo CNTC. Apesar desse documento não ser destinado ao ACE e nem ter como referência a temática da dengue, foi incluído na análise por ter sido citado na literatura como importante política de educação profissional, tendo o ACE como integrante do grupo profissional que constitui os agentes de vigilância em saúde.

Outra política que se destacou no âmbito do Ministério da Saúde foi o Programa de Formação de Agentes Locais de Vigilância em Saúde/Proformar (14). Essa foi uma ação de abrangência nacional, conjugando as modalidades presenciais e a distância. O programa formou 31.427 trabalhadores da saúde no ano de 1999.

No que tange à bibliografia utilizada pelos documentos, observou-se que apenas dois não incluíram referências. A maior parte, entretanto, adotou um referencial amplo, atualizado e de diferentes instituições e idiomas. Desses, todos empregaram as normas da ABNT.

\section{Considerações finais}

A partir da análise dos documentos oficiais que compuseram o corpus da pesquisa, observou-se que a política de formação direcionada aos ACE, no contexto específico da dengue, vem sendo negligenciada pelas principais agências governamentais. Pouco espaço e atenção é dado às ações de educação direcionada aos ACE sem menção às formas permanentes e interativas dos espaços formais e não formais de ensino, tanto nos serviços de saúde quanto nas comunidades e demais territórios.

No âmbito das políticas de educação, antes da Constituição Federal de 1988, os cursos destinados aos trabalhadores técnicos da saúde eram criados e regulamentados exclusivamente pelo Ministério da Educação. Assim, na década de 1990, ao constar na Constituição a responsabilização da formação dos trabalhadores do SUS, pelo Ministério da Saúde, este passou a integrar as formulações e regulamentações dos cursos da área da saúde.

Para o exercício da função de ACE não há exigências mais complexas de formação ou experiência. De acordo com a CBO e a Lei 11350/2006, o único requisito para ser ACE é o de possuir o ensino fundamental completo. Assim, o processo de constituição da categoria profissional foi delineado por trabalhadores de diversos níveis e áreas de formação. Em decorrência do cenário nebuloso das arboviroses contemporâneas é fato que uma formação inexistente ou inadequada é inconcebível. É importante considerar que se não há uma formação sólida, não há possibilidades de transformação desse quadro cíclico da doença, com todos os determinantes que a constituem.

Constata-se, a partir da análise descritiva dos documentos, que as políticas de formação dos ACE sobre a dengue não foram instituídas. Identifica-se ainda o fato de eles serem imbuídos de atividades que exigem muito mais conhecimentos e escolaridade que garantido pelas políticas. Há indicações nos documentos 
para que os ACE orientem a população em ações de 'educação em saúde'. Contudo, como é possível realizar na prática um trabalho bem fundamentado sem antes ter recebido uma formação para tal? É notório que as recorrentes epidemias de dengue clamam por profissionais mais bem formados e essa condição não pode passar de forma latente aos olhos das políticas de recursos humanos de saúde no Brasil e no mundo.

Do ponto de vista da formação dos ACE, a implementação efetiva das medidas de promoção da saúde, prevenção e controle da dengue exige trabalhadores qualificados, além de outros fatores como equipamentos e infraestruturas apropriadas. Observa-se, contudo, que pouco espaço é dado às formulações de políticas e programas de educação e formação elaborados pelo poder público destinados aos ACE.

Assim, a formação dos ACE se dá quase predominantemente nas práticas cotidianas do trabalho/ serviço. Nesses termos, em sua gênese, a vigilância em saúde, em especial, a formação dos ACE está sendo constituída como um serviço estritamente operacional e técnico, sem aprofundamento teórico e prático para tal. Em última instância, torna-se necessário refletir e aprofundar a discussão sobre quem é o ACE e o motivo pelo qual os cursos técnicos, bem como a regulamentação da profissão não constam nas pautas principais das políticas de controle de vetores.

Ações políticas mais integradas e intersetoriais podem auxiliar na legitimação das práticas de formação e trabalho do ACE, auxiliando-o a se reconhecer como pertencente a uma categoria profissional, com espaço específico para sua organização e mobilização político-institucional. E, o mais importante, a institucionalização de uma sólida política de educação profissional direcionada ao ACE pode contribuir para um avanço significativo na prevenção e no controle da doença e vetores.

Assim, considerando que as ações de vigilância foram descentralizadas e que o Brasil tem mais de cinco mil municípios, pesquisas futuras tornam-se necessárias para investigação de políticas de formação dos ACE nos estados e municípios brasileiros. Embora exista uma política incipiente de formação de trabalhadores do setor público em vigilância em saúde, permanece a ausência de regulamentação mais sólida do trabalho do ACE, bem como sobre a sua formação. Todos esses aspectos precisam ser pensados e aprofundados em futuros estudos para se delinear uma política pública de saúde para a formação do agente de combate às endemias de forma integral e plena.

\section{Referências}

1. Pimenta Júnior FG. Gestão e planejamento na prevenção e no controle da dengue. In: Cunha, RV, Pimenta DN, Valle D, organizadores. Dengue: teorias e práticas. Rio de Janeiro: Fiocruz; 2015.

2. Costa, SM, Araújo, FF, Martins, LV, Nobre LLR, Araújo FM, Rodrigues CAQ. Agente comunitário de saúde: elemento nuclear das ações em saúde. Ci Saúde Coletiva. 2013;18(7):2147-56.

3. Martins CM, Stauffer AB. Sobre a produção da sociabilidade capitalista: o discurso sobre trabalho, comunicação e participação nos manuais dos agentes comunitários de saúde1. Reciis [Internet]. 2012 dez. [citado em 2016 set. 20];6(4). Disponível em: http://www.reciis.icict.fiocruz .br/index.php/reciis/ article/view/582. Acesso em: 20 set. 2016.

4. Marzari CK, Jungues JR, Selli L. Agentes comunitários de saúde: perfil e formação. Ci Saúde Coletiva. 2011;16(supl. 1):120-35.

5. Morosini MV. Educação e trabalho em disputa no SUS: a política de formação dos agentes comunitários de saúde. Rio de Janeiro: EPSJV; 2010.

6. Oliveira Junior JG. Agentes comunitários de saúde: fatores restritivos e facilitadores do seu trabalho na Estratégia de Saúde da Família. Dissertação. Rio de Janeiro: Escola Nacional de Saúde Pública Sergio Arouca; 2012.

7. Queirós AAL, Lima LP. A institucionalização do trabalho do agente comunitário de saúde. Trab Educ Saúde. 2012 out.;10(2):257-81. 
8. Maia LS, Silva OA, Rodrigues PM, Barros MRB, Araújo AM. Conhecimentos dos agentes de endemias: aplicação de bases teóricas sobre saneamento e ambiente. R Visa em Debate: Soc Ci \& Tec. 2013;1(3):27-34.

9. Fraga LS, Monteiro S. A gente é um passador de informação: práticas educativas dos agentes de combate a endemias no serviço de controle de zoonoses em Belo Horizonte MG, Brasil. Soc Saúde. [Internet]. 2014 [citado em 2017 fev. 20];23(3):993-1006. Disponível em: http://www. scielo.br/scielo. php?pid=S0104-12902014000300993\&script=sci abstract\&tlng=pt.

10. Batistella CEC. Qualificação e identidade profissional dos trabalhadores técnicos da vigilância em saúde: entre ruínas, fronteiras e projetos. In: Morosini MVG, Lopes MCR, Chagas DC, Chinelli F, Vieira M, organizadores. Trabalhadores técnicos da saúde: aspectos da qualificação profissional nos SUS. Rio de Janeiro: EPSJV; 2013.

11. Torres R. Agentes de combate a endemias: a construção de uma identidade sólida e a formação ampla em vigilância são desafios dessa categoria. R Poli: Saúde Educ Trab. 2009 jan./fev.;(3):16-17.

12. Brasil. Casa Civil. Constituição da República Federativa do Brasil de 1988 [Internet]. Brasília; 1998 [citado em 2017 fev. 20]. Disponível em: http://www.planalto.gov.br/ccivil 03/constituição / constituicaocompilado.htm.

13. Hochman G. Reformas, instituições e políticas de saúde no Brasil (1930-1945). Educar. 2005;(25):127-41.

14. Maar WL. O que é política. 20. ed. São Paulo: Brasiliense; 1982.

15. Fleury, S; Ouverney, AM. Política de saúde: uma política social. In: Giovanella L, Sarah E, Lobato LVC, organizadores. Políticas e sistema de saúde no Brasil. Rio de Janeiro: Fiocruz; 2008.

16. Pimenta DN. Determinação social, determinantes sociais da saúde e a dengue: caminhos possíveis. In: Cunha RV, Pimenta DN, Valle D, organizadores. Dengue: teorias e práticas. Rio de Janeiro: Fiocruz; 2015.

17. Brasil. Ministério da Saúde. Portaria no 1.007, 4 maio. 2010. Define critérios para regulamentar a incorporação do agente de combate às endemias (ACE), ou dos agentes que desempenham essas atividades, mas com outras denominações, na atenção primária à saúde para fortalecer as ações de vigilância em saúde junto às equipes de saúde da família. Brasília; 2010.

18. Brasil. Lei n. 11.350, de 5 out. 2006. Regulamenta as atividades e formas de contratação dos agentes comunitários de saúde e agentes de combate às endemias. Diário Oficial da República Federativa do Brasil, Brasília; 6 out. 2006.

19. Brasil. Ministério da Saúde. Portaria no 1.399, de 15 dez. 1999. Estabelece procedimentos para elaboração, implementação e acompanhamento da Programação Pactuada e Integrada de Vigilância em Saúde - PPI-VS. Brasília; 1999.

20. Brasil. Ministério do Trabalho e Emprego. Classificação Brasileira de Ocupações. Brasília; 2015.

21. Franzoi NL. Entre a formação e o trabalho: trajetórias e identidades profissionais. Porto Alegre: UFRGS; 2006.

22. Brasil. Lei n. 12.994, de 17 jun. 2014. Institui piso salarial profissional nacional e diretrizes para o plano de carreira dos Agentes Comunitários de Saúde e dos Agentes de Combate às Endemias. Diário Oficial da República Federativa do Brasil, Brasília; 18 jul. 2014.

23. Minayo MCS. O desafio do conhecimento: pesquisa qualitativa em saúde. 13. edição. São Paulo: Hucitec; 2013.

24. Pimentel A. O método da análise documental: seu uso numa pesquisa historiográfica. Cad Pesq [Internet]. 2001 [citado em 2017 fev. 20];(114):179-95.

25. Silva JRS, Almeida CD, Guindani JF. Pesquisa documental: pistas teóricas e metodológicas. R Bras Hist Ci Soc. 2009 jul.;1(1):1-15.

26. Bardin L. Análise de conteúdo. Lisboa: Edições 70; 2011.

27. Franco ML Análise de conteúdo. 4. ed. Brasília: Liber Livro, 2012.

28. Figueiro AC, Sóter AP, Braga C, Hartz ZMA, Samico I. Análise da lógica de intervenção do Programa Nacional de Controle da Dengue. R Bras Saúde Matern Infantil [Internet]. 2010 [citado em 201720 fev.];10(suppl.1):s93-s106.

29. Brasil. Ministério da Educação, Secretaria de Educação Profissional e Tecnológica. Guia Pronatec de cursos de formação inicial e continuada ou qualificação profissional: agente de combate a endemias. 4. ed. Brasília; 2016. 
30. Brasil. Ministério da Saúde. Secretaria de Vigilância em Saúde. Secretaria de Atenção à Saúde O agente comunitário de saúde no controle da dengue. Brasília; 2009.

31. Brasil. Ministério da Saúde. Secretaria de Atenção à Saúde. Departamento de Atenção Básica. Vigilância em saúde: dengue, esquistossomose, hanseníase, malária, tracoma e tuberculose. 2. ed. rev. Brasília; 2008. 195 p. (Série A. Normas e Manuais Técnicos). (Cadernos de Atenção Básica, n. 21).

32. Brasil. Ministério da Saúde. Secretaria de Vigilância em Saúde. Departamento de Vigilância Epidemiológica. Diretrizes nacionais para prevenção e controle de epidemias de dengue. Brasília; 2009. 160 p. (Série A. Normas e Manuais Técnicos).

33. Brasil. Ministério da Saúde. Fundação Nacional de Saúde. Plano Nacional de Controle da Dengue (PNCD). Brasília; 2002.

34. Brasil. Ministério da Saúde. Secretaria de Vigilância em Saúde. Programa nacional de controle da dengue amparo legal à execução das ações de campo: imóveis fechados, abandonados ou com acesso não permitido pelo morador. 2. ed. Brasília; 2006.

35. Brasil. Ministério da Saúde. Secretaria de Gestão do Trabalho e da Educação na Saúde. Departamento de Gestão da Educação na Saúde. Técnico em vigilância em saúde: diretrizes e orientações para a formação. Brasília; 2011. 72 p. (Série A. Normas e Manuais Técnicos).

36. Brasil. Ministério da Saúde. Fundação Nacional de Saúde. Dengue, instruções para pessoal de combate ao vetor: manual de normas técnicas. 3. ed., rev. Brasília; 2001.

37. Fundação Oswaldo Cruz. Nota técnica no 2/2011/IOC-Fiocruz/Diretoria. Rio de Janeiro: IOC; 2011.

38. Fundação Oswaldo Cruz. Nota técnica No 3/2014/IOC-Fiocruz/Diretoria. Rio de Janeiro: IOC; 2014.

39. Brasil, Ministério da Educação. Instituto Federal de Educação Técnica e Tecnológica do Rio Grande do Norte. Projeto Pedagógico do Curso de Formação Inicial e Continuada ou Qualificação Profissional em Agente de Combate às Endemias na modalidade presencial no âmbito do Pronatec Eixo Tecnológico: Ambiente e Saúde. Natal, 2013.

40. Brasil. Ministério Educação. Secretaria de Educação Profissional e Tecnológica. Catálogo nacional de cursos técnicos e tecnológicos/agentes de vigilância em saúde. 3. ed. Brasília; 2016.

41. Brasil. Ministério da Educação. Instituto Federal de Educação Técnica e Tecnológica do Paraná. Formação inicial e continuada/agentes de saúde: Pronatec. Curitiba; 2012.

42. Reis JS. Formação profissional do agente de vigilância em saúde: análise da proposta de Formação do Programa Proformar [dissertação]. Rio de Janeiro: Escola Politécnica de Saúde Joaquim Venâncio; 2013.

43. Gondim GMM, Monken M. Saúde, educação, cidadania e participação: a experiência do Proformar. Trab Educ Saúde. 2003 set.;1(2);335-53.

44. Fonseca AF. Sobre o trabalho e a formação de agentes de saúde em tempos de Zika. Trab Educ Saúde [Internet]. 2016 maio/ago.;14(2):327.

45. Pimenta DN. A (des)construção da dengue: de tropical a negligenciada. In: Cunha RV, Pimenta DN, Valle D, organizadores. Dengue: teorias e práticas. Rio de Janeiro: Fiocruz; 2015. 